\title{
Two new species of Urocleidoides Mizelle et Price, 1964 (Monogenoidea) from the gill lamellae of profundulids and poeciliids from Central America and southern Mexico
}

\author{
Edgar F. Mendoza-Franco ${ }^{1}$, Juan Manuel Caspeta-Mandujano ${ }^{2}$, Guillermo Salgado-Maldonado ${ }^{3}$ and Wilfredo \\ Antonio Matamoros ${ }^{4}$ \\ ${ }^{1}$ Instituto de Ecología, Pesquerías y Oceanografía del Golfo de México (EPOMEX), Universidad Autónoma de Campeche, \\ Campeche, México; \\ ${ }^{2}$ Facultad de Ciencias Biológicas y Centro de Investigaciones Biológicas, Universidad Autónoma del Estado de Morelos, \\ Cuernavaca, Morelos, Mexico; \\ ${ }^{3}$ Instituto de Biología, Universidad Nacional Autónoma de México, Mexico, D. F., Mexico; \\ ${ }^{4}$ Facultad de Ciencias Biológicas, Universidad de Ciencias y Artes de Chiapas, Tuxtla Gutiérrez, Chiapas, Mexico
}

\begin{abstract}
During investigations of gill ectoparasites (Platyhelminthes) parasitising freshwater fish from Central America (Guatemala, Honduras, El Salvador and Panama) and southeastern Mexico (Guerrero, Oaxaca and Chiapas), the following dactylogyrid monogenoidean were found: Urocleidoides simonae sp. n. from Profundulus punctatus (Günther) (type host), Profundulus balsanus Ahl, Profundulus guatemalensis (Günther), Profundulus kreiseri Matamoros, Shaefer, Hernández et Chakrabarty, Profundulus labialis (Günther), Profundulus oaxacae (Meek), Profundulus sp. 1 and Profundulus sp. 2 (all Profundulidae); Urocleidoides vaginoclaustroides sp. n. from Pseudoxiphophorus bimaculata (Heckel) (type host) and Poeciliopsis retropinna (Regan) (both Poeciliidae); and Urocleidoides vaginoclaustrum Jogunoori, Kritsky et Venkatanarasaiah, 2004 from P. labialis, Profundulus portillorum Matamoros et Shaefer and Xiphophorus hellerii Heckel (Poeciliidae). Urocleidoides simonae sp. $\mathrm{n}$. differs from all other congeneric species in having anchors with well-differentiated roots, curved elongate shaft and short point. Urocleidoides vaginoclaustroides sp. n. most closely resembles $U$. vaginoclaustrum, but differs from this species mainly in the shape of its anchors (i.e. evenly curved shaft and short point $v S$ curved shaft and elongate point extending just past the tip of the superficial anchor root). The complexity of potential hosts for species of Urocleidoides and their effect on its distribution on profundulid and poeciliid fishes are briefly discussed.
\end{abstract}

Keywords: Dactylogyridae, Profundulus, Pseudoxiphophorus, Poeciliopsis, Xiphophorus, Guerrero, Oaxaca, Chiapas, Guatemala, Honduras, El Salvador, Panama

This article contains supporting information (Tables S1, S2) online at http://folia.paru.cas.cz/supp1/2015-62-059.pdf

Urocleidoides Mizelle et Price, 1964 (sensu Kritsky et al. 1986) (Monogenoidea: Dactylogyridae) currently contains up to 20 accepted species, which exhibit a broad host specificity on Neotropical freshwater fishes of different genera, including Brachyhypopomus Mago-Leccia (Hypopomidae), Characidium Reinhardt (Characidae), Ctenolucius Gill (Ctenoluciidae), Curimata Bosc (Curimatidae), Hoplias Gill (Erythrinidae), Hypopomus Gill (Hypopomidae), Piabucina Valenciennes (Lebiasinidae), Poecilia Bloch et Schneider, Rhytiodus Kner (Anostomidae), Saccodon Kner (Parodontidae) and Xiphophorus Heckel (Poeciliidae) from Argentina, Brazil, Colombia, Panama and Trinidad (Kritsky et al. 1986, Moreira et al. 2015). Although species of Urocleidoides have a broad distribution in the tropics, there are still few published studies documenting monogenoidean diversity in host species from these 10 families.

During a study on fish ectoparasites found in rivers from Central America (Guatemala, Honduras, El Salvador and Panama) and southeastern Mexico (Guerrero, Oaxaca and Chiapas), two undescribed species of Urocleidoides were discovered on the gill lamellae of species of Profundulus (Miller) (Profundulidae), Pseudoxiphophorus bimaculata (Heckel) and Poeciliopsis retropinna (Regan) (both Poeciliidae). An additional species was also identified as Urocleidoides vaginoclaustrum Jogunoori, Kritsky et Venkatanarasaiah, 2004 from Profundulus portillorum Matamoros et Shaefer, P. labialis (Günther) and Xiphophorus hellerii Heckel. 
Herein, the new species are described, parameters of infection as well as new morphometric data and supplemental observations for $U$. vaginoclaustrum are provided.

\section{MATERIALS AND METHODS}

Fish specimens were captured from June 2012 to May 2014 from 10 localities in Central American countries (Guatemala, Honduras, El Salvador and Panama) and 10 localities in the states of Guerrero, Oaxaca and Chiapas in Mexico using an electrofishing device and deep-nets, transported alive to the laboratory and examined for monogenoideans within 24 hours post-capture (see Salgado-Maldonado et al. 2014). The collection methods, preparation and study of monogenoideans found on profundulid and poeciliid fishes follow Mendoza-Franco et al. (2013).

Measurements are given in micrometres $(\mu \mathrm{m})$ and correspond to the straight-line distance between the extreme points of structures. All measurements are expressed as the mean, followed by the range and number (n) of structures measured in parentheses. Body length measurements include the haptor. Type and voucher specimens of helminths were deposited in the Institute of Parasitology, České, Budějovice, Czech Republic (IPCAS) and the Colección Nacional de Helmintos, Institute of Biology, National Autonomous University of Mexico, Mexico (CNHE).

For comparison, the following specimens were examined: 3 paratypes of $U$. vaginoclaustrum (Natural History Museum, London-BMNH 2003.9.14.13-20) and 6 paratypes of Urocleidoides flegomai Mendoza-Franco, Aguirre-Macedo et Vidal-Martinez, 2007 (IPCAS M-433). Definitions of ecological terms are those suggested by Bush et al. (1997). The scientific names of the hosts are those provided by Doadrio et al. (1999) and host body lengths are expressed as total length (TL) in $\mathrm{mm}$.

\section{RESULTS}

Class Monogenoidea Bychowsky, 1937

Subclass Polyonchoinea Bychowsky, 1937

Order Dactylogyridea Bychowsky, 1937

Dactylogyridae Bychowsky, 1933

Urocleidoides simonae sp. $\mathrm{n}$.

Figs. 1-10

ZooBank number for species:

urn:1sid:zoobank.org:2DBB4F1E-054E-4391-9267-AE97594372DC

Diagnosis (based on 32 stained specimens with Gomori's trichrome and on 6 specimens remounted from a mixture of lactic-acid and glycerin-ammonium picrate to Canada balsam - see Mendoza-Franco et al. 2013): Body fusiform, greatest width near level of gonads. Cephalic lobes moderately developed; 3 bilateral pairs of head organs; cephalic glands indistinct. Eyespots 4, subequal in size, members of respective pairs equidistant. Pharynx subovate. Peduncle broad, tapered posteriorly; haptor subtrapezoidal. Anchors similar in shape, each with well-differentiated roots, curved elongated shaft, short point (shaft and point of ventral anchor extending poste- riorly from haptor). Ventral bar from straight to slightly arched on its medial portion with notable expanded ends. Dorsal bar broadly V-shaped. Hooks similar in shape, each with protruding thumb, delicate shaft and point, dilated shank; hook pair 1, 5 reduced in size; filamentous hooklet loop nearly $50 \%$ of shank length. Male copulatory organ (MCO) a coiled tube of about 4-5 counterclockwise rings with spherical base surrounded by lateral flange. Accessory piece comprising a sheath along distal shaft of the MCO. Vaginal pore sinistral, submarginal; vaginal canal distally coiled; seminal receptacle subspherical, lying on anterior margin of the germarium. Gonads overlapping. Testis dorsal, slightly visible at end of germarium; proximal portion of vas deferens not observed; seminal vesicle a V-shaped dilation (located right below of the $\mathrm{MCO}$ ) of distal vas deferens; prostatic reservoir lying posterior to base of $\mathrm{MCO}$; oviduct, ootype and uterus not observed. Vaginal sclerite composed of grooved rod with distal hook, subterminal short projection. Vitellarium dense, coextensive with gut. Measurements from different hosts and localities provided in Table 1.

Ty pe host: Profundulus punctatus (Günther) (Teleostei: Profundulidae).

Type 1 o c a lity: Río Nandalumi, Chiapa de Corso, Chiapas, Mexico (16² $43^{\prime} 18^{\prime \prime} \mathrm{N}$; 92 $58^{\circ} 52^{\prime \prime} \mathrm{W}$; altitude $\left.486 \mathrm{~m}\right), 28$ May 2013.

Prevalence and mean intensity of infection: 25 fish (mean TL $54 \mathrm{~mm}$ ) infected of 31 examined (81\%), mean intensity 13.4 , range $2-30$ worms per infected fish.

Site of infection: Gill lamellae.

Other localities in Chiapas Mexico and parameters of infection: Río at rancho San Antonio, Chicoasén, Chiapas (16 58 '31"'N; 9303'44"W; 375 m), 6 fish (mean TL $56 \mathrm{~mm}$ ) infected of 8 examined (75\%), mean intensity 17.6, range 1-66 worms per infected fish. Arroyo Ojo de Agua, El Canelar, La Frailesca, Chiapas (16²32'08"N; $\left.92^{\circ} 55^{\prime} 03^{\prime \prime W} ; 466 \mathrm{~m}\right), 5 / 7$ (mean TL $61 \mathrm{~mm}$ ) (71\%), 14 (worms not measured), 2-46 (see Table 1).

Other hosts in Mexican localities: Guerrero state - Profundulus balsanus Ahl from Río La Laca (Río Papagayo basin) $\left(17^{\circ} 14^{\prime} 09^{\prime \prime N}\right.$; 98³9'56"W; $\left.1394 \mathrm{~m}\right), 21 / 30$ (mean TL $57 \mathrm{~mm})(70 \%), 3.4,1-11$, March 2013. Río Cahoapan $\left(17^{\circ} 16^{\prime} 38^{\prime \prime} \mathrm{N} ; 99^{\circ} 35^{\prime} 05^{\prime \prime} \mathrm{W} ; 422 \mathrm{~m}\right.$ ), 11/30 (mean TL $72 \mathrm{~mm}$ ) (37\%), 1.4, 1-2, March 2013. Río Tamarindo (Río Nexpa basin) (17\%00'37"N; 9906'01"W; 417 m), 23/30 (mean TL $55 \mathrm{~mm}$ ) (77\%), 9.7 (worms not measured), 2-28, March 2013 (see Table S1). Oaxaca state - Profundulus oaxacae (Meek) from Creek Los Sabinos (Río Atoyac-Verde basin) (16 $\left.25^{\prime} 40^{\prime \prime} \mathrm{N} ; 97^{\circ} 04^{\prime} 29^{\prime \prime} \mathrm{W} ; 1106 \mathrm{~m}\right), 4 / 30$ (mean TL $54 \mathrm{~mm}$ ) (13\%), 1.7, 1-4, February 2013. Profundulus sp. 1 from Río Chicaguaxtla, Concepción del Progreso (Atoyac-Verde basin) $\left(17^{\circ} 03^{\prime} 30^{\prime \prime} \mathrm{N} ; 97^{\circ} 51^{\prime} 33^{\prime \prime} \mathrm{W} ; 867 \mathrm{~m}\right), 20 / 31$ (mean TL $56 \mathrm{~mm})(64 \%), 4.3,1-10$, February 2013. Profundulus sp. 2 from Río Chico, San Lorenzo Albarradas (Atoyac-Verde basin) $\left(16^{\circ} 55^{\prime} 35^{\prime \prime} \mathrm{N} ; 96^{\circ} 12^{\prime} 27^{\prime \prime} \mathrm{W} ; 1316 \mathrm{~m}\right), 20 / 30$ (mean TL $63.8 \mathrm{~mm}$ ) (67\%), 4.5, 1-22, February 2013. Profundulus balsanus from Río del Aguacate, Juquilá, Río Manialtépec basin (1607'19"N; 9708'23"W; $482 \mathrm{~m}$ ), 15/31 (mean TL $56.9 \mathrm{~mm}$ ) (48\%), 6.4, 2-18, February 2013 (see Table S2). Chiapas state - Profundulus labialis (Günther) from Río at rancho San An-

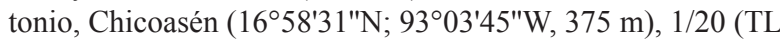




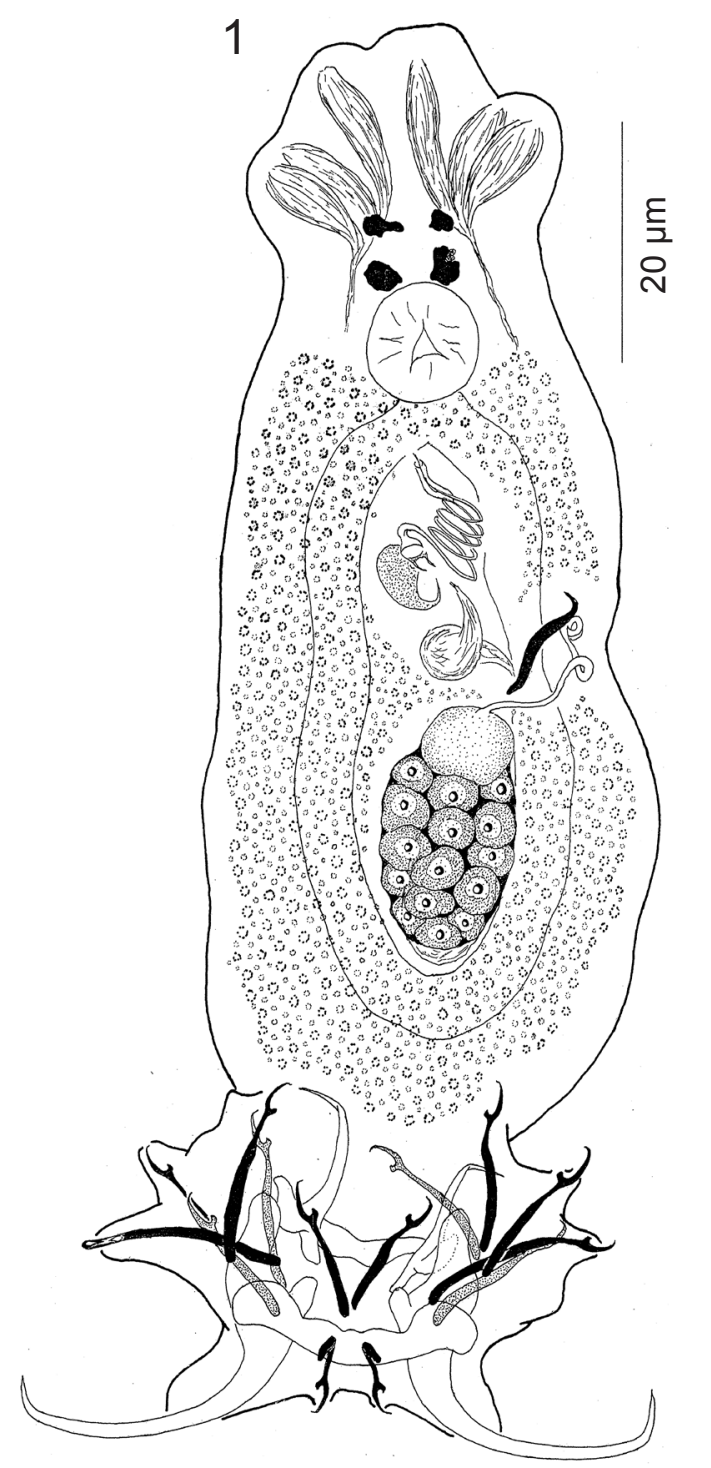

2
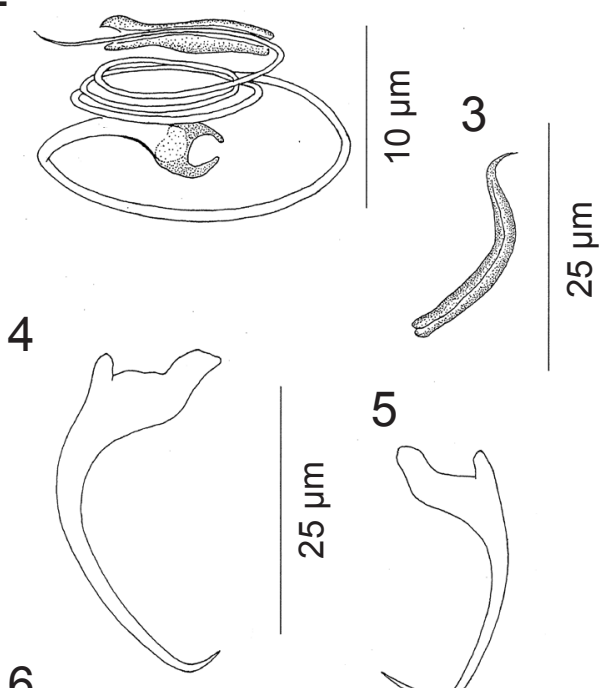

5

6
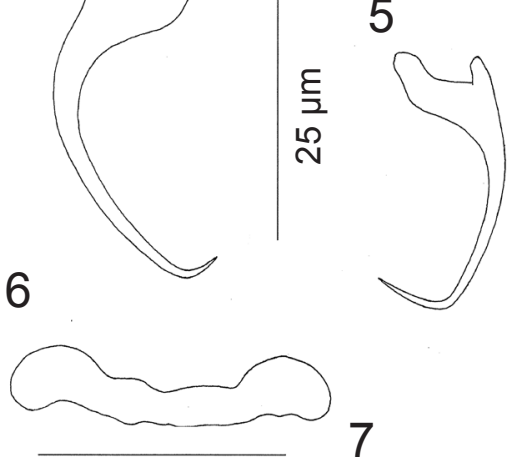

\section{7}

8
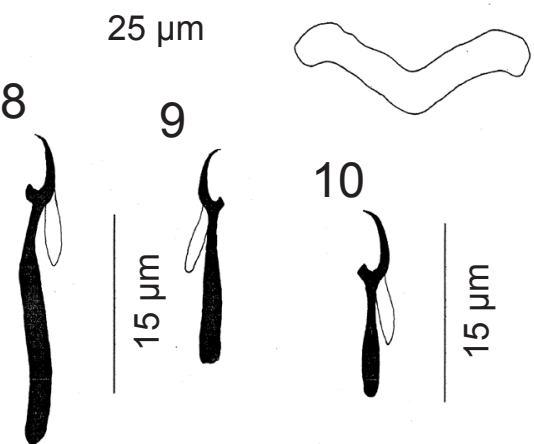

Figs. 1-10. Urocleidoides simonae sp. n. from Profundulus punctatus (Günther) from Río Nandalumi, Chiapas, Mexico. Fig. 1. Composite (ventral view). Fig. 2. Copulatory complex (ventral). Fig. 3. Vaginal sclerite. Fig. 4. Ventral anchor. Fig. 5. Dorsal anchor. Fig. 6. Ventral bar. Fig. 7. Dorsal bar. Fig. 8. Hook (pair 2). Fig. 9. Hook (pair 1). Fig. 10. Hook (pair 5).

Table 1. Comparative measurements of Urocleidoides simonae sp. n. (Monogenoidea) from the gill lamellae of two species of Profundulus Miller (Profundulidae) from different localities in the state of Chiapas, Mexico.

\begin{tabular}{|c|c|c|c|c|c|}
\hline & \multicolumn{2}{|c|}{ Profundulus punctatus (Günther)* } & \multicolumn{3}{|c|}{ Profundulus labialis (Günther) } \\
\hline & Río Nandalumi† & Río Chicoasen & Río Nandalumi & Río Chicoasen & Creek Copainalá \\
\hline Body length & $154(115-205 ; \mathrm{n}=19)$ & $149(93-215 ; \mathrm{n}=18)$ & $163(115-205 ; \mathrm{n}=19)$ & $142(75-210 ; \mathrm{n}=8)$ & $141(105-170 ; n=9)$ \\
\hline Greatest width & $55(34-65 ; n=12)$ & $44(26-55 ; n=10)$ & $50(38-63 ; n=18)$ & $48(42-55 ; n=4)$ & $51(42-62 ; \mathrm{n}=8)$ \\
\hline Pharynx & $13(12-15 ; n=4)$ & - & - & - & - \\
\hline Haptor width & $47(38-60 ; n=24)$ & $54(42-67 ; \mathrm{n}=13)$ & $50(40-70 ; \mathrm{n}=19)$ & $51(40-65 ; n=9)$ & $50(38-65 ; \mathrm{n}=11)$ \\
\hline Ventral anchor length & $32(30-33 ; \mathrm{n}=40)$ & $31(30-33 ; \mathrm{n}=22)$ & $31(29-33 ; \mathrm{n}=26)$ & $29(25-32 ; n=16)$ & $30(27-32 ; \mathrm{n}=19)$ \\
\hline Base width & $12(11-13 ; \mathrm{n}=19)$ & $11(11-12 ; \mathrm{n}=10)$ & $11(10-12 ; \mathrm{n}=18)$ & $10(9-11 ; n=14)$ & $11(10-12 ; \mathrm{n}=13)$ \\
\hline Dorsal anchor length & $26(25-27 ; \mathrm{n}=37)$ & $26(24-28 ; \mathrm{n}=20)$ & $26(24-29 ; \mathrm{n}=27)$ & $21(20-23 ; n=11)$ & $25(23-27 ; \mathrm{n}=19)$ \\
\hline Base width & $11(10-11 ; \mathrm{n}=8)$ & $10(10-11 ; \mathrm{n}=8)$ & $10(9-11 ; n=13)$ & $10(9-10 ; \mathrm{n}=8)$ & $10(10-11 ; \mathrm{n}=11)$ \\
\hline Ventral bar length & $28(25-31 ; \mathrm{n}=19)$ & $28(26-30 ; n=15)$ & $26(25-28 ; n=18)$ & $23(21-25 ; n=5)$ & $25(21-28 ; \mathrm{n}=10)$ \\
\hline Dorsal bar length & $20(18-21 ; \mathrm{n}=10)$ & $20(18-22 ; \mathrm{n}=8)$ & $19(18-21 ; n=6)$ & $22(20-23 ; n=3)$ & $18(\mathrm{n}=1)$ \\
\hline Hook pair 1 & $15(\mathrm{n}=2)$ & $14(14-15 ; \mathrm{n}=9)$ & $14(14-15 ; n=5)$ & $13(11-14 ; n=7)$ & $13(13-15 ; n=4)$ \\
\hline Hook pair 5 & $10(10-11 ; n=4)$ & $10(10-11 ; \mathrm{n}=68)$ & $10(\mathrm{n}=3)$ & - & $10(\mathrm{n}=3)$ \\
\hline Hook pairs $2-4,6$ and 7 & $20(19-22 ; n=9)$ & $21(18-22 ; \mathrm{n}=9)$ & $20(18-21 ; n=8)$ & $18(16-20 ; \mathrm{n}=8)$ & $18(\mathrm{n}=2)$ \\
\hline First ring of the $\mathrm{MCO}$ & $11(10-12 ; n=3)$ & $13(11-16 ; n=7)$ & $12(10-13 ; n=6)$ & $142(75-210 ; \mathrm{n}=8)$ & $13(12-14 ; n=5)$ \\
\hline Germarium length & $24(16-28 ; n=6)$ & - & $30(25-35 ; n=6)$ & - & - \\
\hline Germarium width & $14(12-17 ; n=6)$ & - & $13(12-16 ; n=5)$ & - & - \\
\hline Testis length & - & - & - & - & - \\
\hline Testis width & - & - & - & - & - \\
\hline Vaginal sclerite length & $11(10-13 ; \mathrm{n}=16)$ & $12(11-13 ; n=7)$ & $11(10-12 ; n=9)$ & $11(\mathrm{n}=3)$ & - \\
\hline
\end{tabular}

\footnotetext{
$*=$ type host; $\uparrow=$ type locality.
} 
$25 \mathrm{~mm})(5 \%), 9$; Creek Tres Picos, Copainalá (1703'28"N; 9311'51"W; $325 \mathrm{~m}$ ), 6/30 (mean TL $52.5 \mathrm{~mm}$ ) (20\%), 14, 3-35; and Río Nandalumi, Chiapa de Corso, 1/16 (TL 42 mm) (6\%), 23, May 2013 (see Table 1).

Other hosts, localities, parameters of infection and dates in Central American localit i es: Guatemala - Profundulus guatemalensis (Günther) from Río Nil (Río Guacalate basin) (14³3'54"N; 9143'25"W; 237 m), 30/30 (mean TL 53 mm) (100\%), 9, 3-29; Río El Cantil (Río Guacalate basin) $\left(14^{\circ} 21^{\prime} 23^{\prime \prime} \mathrm{N} ; 90^{\circ} 48^{\prime} 30^{\prime \prime} \mathrm{W} ; 563 \mathrm{~m}\right)$, 26/30 (mean TL $62 \mathrm{~mm}$ ) (87\%), 6.3, 2-25; and Creek El Platanar (Río Guacalate basin) $\left(14^{\circ} 36^{\prime} 59^{\prime \prime N}\right.$; 90 9046'38"W; $1628 \mathrm{~m}$ ), 11/20 (mean TL $52 \mathrm{~mm}$ ) (55\%), 5.2, 1-10, May 2014. El Salvador - Profundulus guatemalensis from Río Cauta (Río Lempa basin) (1346'43"N; 8951'41"W; $591 \mathrm{~m})$, 24/30 (mean TL $52 \mathrm{~mm}$ ) (80\%), 4.4, 1-14 and Río Cauca (Río Lempa basin) (1346'42"N; 8946'42"W; 450 m), 29/30 (mean TL $56 \mathrm{~mm}$ ) (97\%), 9, 2-33, May 2014. Profundulus kreiseri Matamoros, Shaefer, Hernández et Chakrabarty from Río Nonoalpa (Río Lempa basin) $\left(14^{\circ} 17^{\prime} 23^{\prime \prime N}\right.$; 8909'11"W; $1040 \mathrm{~m}), 5 / 5$ (mean TL $43 \mathrm{~mm}$ ) (100\%), 8.2, 4-14, May 2014. Quebrada Los Tecomates (Río Lempa basin) $\left(14^{\circ} 18^{\prime} 11^{\prime \prime N}\right.$; $\left.89^{\circ} 09^{\prime} 41^{\prime \prime W} ; 983 \mathrm{~m}\right), 28 / 30$ (mean TL $57 \mathrm{~mm}$ ) (93.3\%), 9.3, 2-36, May 2014.

S pecimen s deposited: Holotype and 21 paratypes in the CNHE (9854 and 9855, respectively), 15 paratypes in IPCAS (M-615); 214 vouchers in CNHE (9856).

Etymology: The species is named after A.R. Simon Saavedra, a wonderful girl that left us unexpectedly at the age of 20 , before fulfilling all her potential as a student of biology.

Differential diagnosis. Based on comparisons of the reproductive organs and copulatory complex morphology, $U$. simonae sp. n. most closely resembles $U$. vaginoclaustroides sp. n. from Pseudoxiphophous bimaculata and U. vaginoclaustrum from Xiphophorus hellerii (Poeciliidae) and Profundulus labialis (present study). The morphology of the copulatory complex and the vagina in these species are similar, differing only in the morphology of anchors (see differential diagnosis for $U$. vaginoclaustroides sp. n.).

Urocleidoides simonae can easily be differentiated from the remaining 20 members of the genus by having anchors with well-differentiated roots, curved elongated shaft and short point (see Figs. 4, 5). Urocleidoides simonae is the species which exhibits the most widespread distribution from Mexico to Central America.

Urocleidoides vaginoclaustroides sp. n. Figs. 11-21

ZooBank number for species:

urn:Isid:zoobank.org:049C041C-D3F9-4224-B2D7-1F8F668BEEFF

Diagnosis (based on 6 stained specimens and 14 specimens remounted from a mixture of lactic-acid and glycerin-ammonium picrate): Body fusiform, greatest width near level of gonads. Cephalic lobes moderately developed; 3 bilateral pairs of head organs; cephalic glands indistinct. Eyespots 4, subequal in size, members of respective pairs equidistant. Pharynx subovate. Peduncle broad, tapered posteriorly; haptor subtrapezoidal. Anchors similar in shape, each with variable and slightly appressed roots, evenly curved shaft and short point. Ventral bar slightly arched on its medial portion with notable expanded ends. Dorsal bar variable, broadly U-shaped with extremities directed laterally. Hooks similar in shape, each with protruding thumb, delicate shaft and point, dilated shank; hook pair 1, 5 reduced in size; filamentous hooklet loop nearly $50 \%$ of shank length. Male copulatory organ (MCO) a coiled tube of about 3-4 counterclockwise rings with spherical base surrounded by lateral flange. Accessory piece comprising a sheath along distal shaft of MCO. Vaginal pore sinistral, marginal; vaginal canal distally coiled; seminal receptacle subspherical, lying on anterior margin of germarium. Gonads overlapping. Testis dorsal, slightly visible at end of germarium; seminal vesicle and route of vas deferens not observed; prostatic reservoir lying posterior to base of MCO; oviduct, ootype, uterus not observed. Vaginal sclerite composed of grooved rod with distal hook, subterminal short projection. Vitellarium dense, coextensive with gut. Measurements from different hosts and localities provided in Table 2.

Type host: Pseudoxiphophorus bimaculata (Heckel) (Cyprinodontiformes: Poeciliidae).

Type locality: Río Danta $\left(16^{\circ} 06^{\prime} 50 " \mathrm{~N} ; 90^{\circ} 56^{\prime} 03^{\prime \prime} \mathrm{W}\right.$ $200 \mathrm{~m}$ ), a tributary of the Río Lacantún basin in the Biosphere Reserve of Montes Azules (BRMA), Chiapas, Mexico, 12 June 2012.

Prevalence and mean intensity of infection: 6 fish (mean TL $61 \mathrm{~mm}$ ) infected of 10 examined (60\%); mean intensity 3.3 worms, range $2-5$ worms per infected fish.

Site of infection: Gill lamellae.

Other records: Arroyo Jose $\left(16^{\circ} 06^{\prime} 50^{\prime \prime} \mathrm{N}\right.$; $90^{\circ} 56^{\prime} 03^{\prime \prime} \mathrm{W}$; $200 \mathrm{~m}$ ), 2/3 (mean TL $51 \mathrm{~mm}$ ) (67\%), 2 (not measured), June 2012. Poeciliopsis retropinna (Regan) from Río Chiriquicito $\left(08^{\circ} 41^{\prime} 14^{\prime \prime N}\right.$; $\left.82^{\circ} 17^{\prime} 27^{\prime \prime} \mathrm{W} ; 1 \mathrm{~m}\right)$ on the western coast of Panama, 2/2 (not measured) (100\%), 9, January 2013 (see Table 2).

$\mathrm{Specimens}$ deposited: Holotype and 10 paratypes in the CNHE (9857 and 9858, respectively), 9 paratypes in IPCAS (M-616); 22 vouchers in the CNHE (9859 and 9860).

E ty mology: The specific name indicates the similarity of this species to Urocleidoides vaginoclaustrum Jogunoori, Kritsky et Venkatanarasaiah, 2004.

Differential diagnosis. The morphometric comparison of anchors of $U$. vaginoclaustroides $\mathrm{sp}$. $\mathrm{n}$. with those of $U$. vaginoclaustrum from Jogunoori et al. (2004), 3 paratypes (BMNH 2003.9.14.13-20) and on specimens of this latter monogenoidean species found in this study (see $U$. vaginoclaustrum below) indicated that both species are different.

Urocleidoides vaginoclaustroides can be differentiated from $U$. simonae and U. vaginoclaustrum by its anchors, which have an evenly curved shaft and short point (curved elongated shaft and short point in U. simonae and "curved shaft and elongate point extending just past the tip of the superficial anchor root" in $U$. vaginoclaustrum) (see Figs. 12-13, 15-16 in the present study and figs. 24-25 in Jogunoori et al. 2004). Additionally, U. vaginoclaustroides slightly differs from $U$. vaginoclaustrum in the length of its anchors (ventral anchor 25-29 $\mu \mathrm{m} v s$ 21-25 $\mu \mathrm{m}$; dor- 

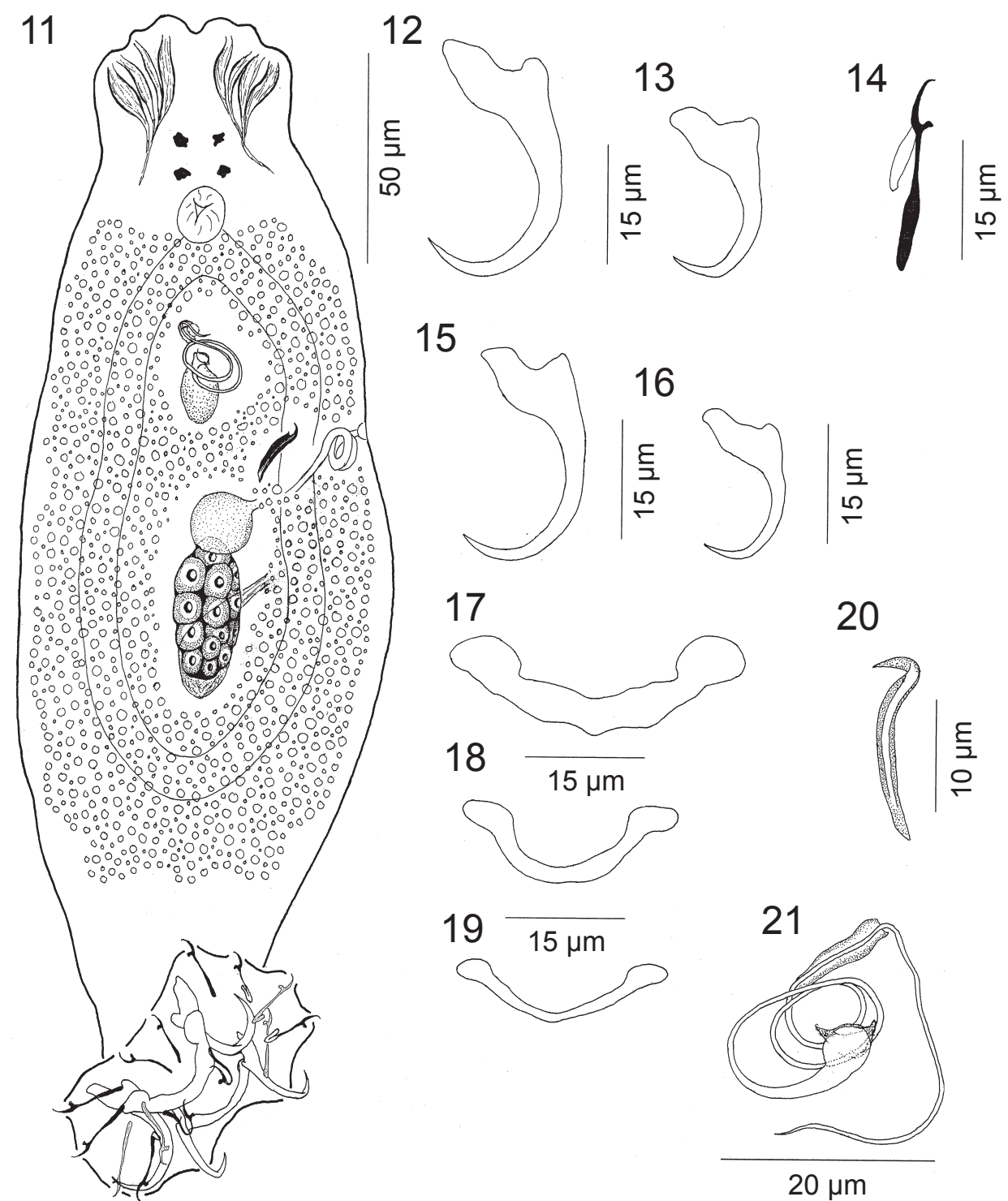

15
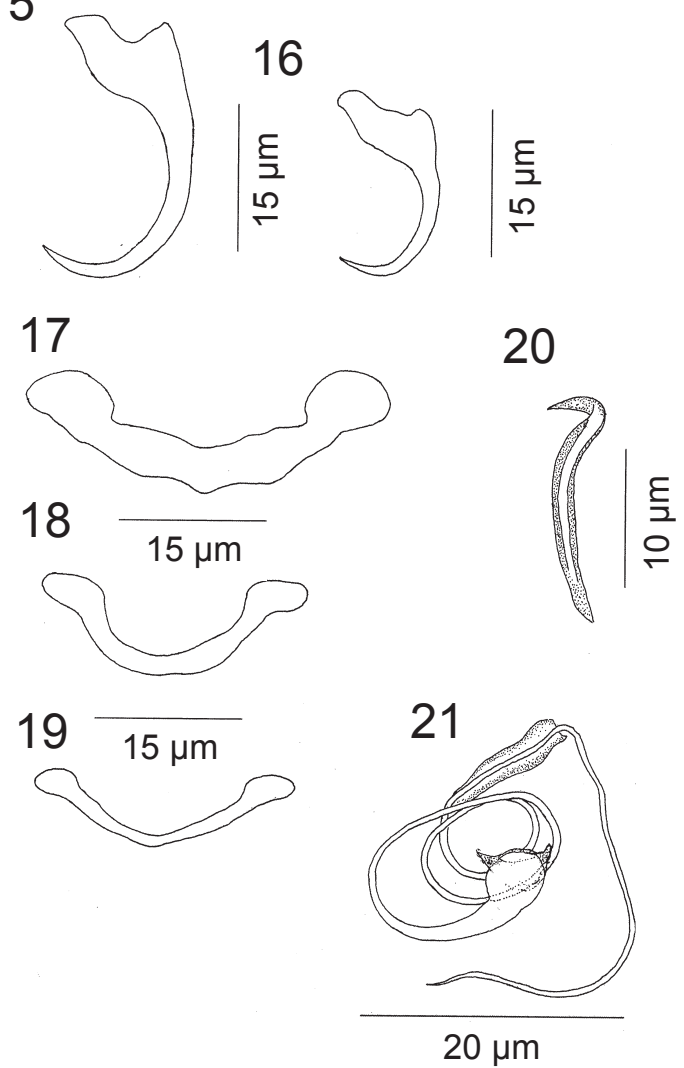

Figs. 11-21. Urocleidoides vaginoclaustroides sp. n. from Pseudoxiphophorus bimaculata (Heckel) from Río Danta, Chiapas, Mexico. Fig. 11. Composite (ventral view). Fig. 12, 15. Ventral anchor. Figs. 13, 16. Dorsal anchor. Fig. 14. Hook (pair 2). Fig. 17. Ventral bar. Figs. 18, 19. Dorsal bars. Fig. 20. Vaginal sclerite. Fig. 21. Copulatory complex (dorsal).

sal anchor 18-22 $\mu \mathrm{m} v s$ 16-19 $\mu \mathrm{m})$ and the length of the vaginal sclerite (13-16 $\mu \mathrm{m} v s$ 16-22 $\mu \mathrm{m})$. Urocleidoides vaginoclaustroides also exhibits other dissimilarities with $U$. vaginoclaustrum, notably its distally coiled vaginal tube (vaginal canal is distally funnel-shaped in U. vaginoclaustrum).

In other features, $U$. vaginoclaustroides shows a slight resemblance to Urocledoides flegomai Mendoza-Franco, Aguirre-Macedo et Vidal-Martínez, 2007 (6 paratypes examined - IPCAS M-433) from Piabucina panamensis Gill from Panama in the general morphology of the vagina, which possesses a coiled tube. Urocleidoides vaginoclaustroides differs from the latter species in the number of rings of the MCO (3-4 rings vs 4-5) and length of the vaginal sclerite (13-16 $\mu \mathrm{m} v s$ 20-25 $\mu \mathrm{m}$ ) and hooks (hook pairs 2, 3, 4 and 7-14-17 $\mu \mathrm{m} v s$ 21-25 $\mu \mathrm{m})$.

The morphometrics of specimens of $U$. vaginoclaustroides from $P$. retropinna collected in Panama did not differ significantly from that of specimens found on $P$. bi- maculata in Mexico. Although the body length of $U$. vaginoclaustroides from $P$. bimaculata varied considerably (i.e. from 157-278), the size of the sclerites is relatively constant among specimens (see Table 2). This variability in body length might be attributable to different degrees of maturity in the worms (see Mendoza-Franco and Vidal-Martínez 2011). Urocleidoides vaginoclaustroides, $U$. simonae as well as $U$. vaginoclaustrum are the only known species of Urocleidoides (sensu stricto) infecting native freshwater host species in Mexico.

Urocleidoides vaginoclaustrum Jogunoori, Kritsky et Venkatanarasaiah, 2004

Measurements of specimens from $X$. hellerii and Profundulus labialis are in Table 2.

Hosts, localities, parameters of infection and collection date: Xiphophorus hellerii Heckel (Cyp- 
Table 2. Comparative measurements of Urocleidoides vaginoclaustroides sp. n. and Urocleidoides vaginoclaustrum Jogunoori, Kritsky et Venkatanarasaiah, 2004 (Monogenoidea).

\begin{tabular}{|c|c|c|c|c|}
\hline & \multicolumn{2}{|c|}{ U. vaginoclaustroides sp. $\mathrm{n}$. from Mexico and Panama } & \multicolumn{2}{|c|}{ U. vaginoclaustrum from Mexico } \\
\hline & $\begin{array}{c}\text { Pseudoxiphophorus bimaculata* } \\
\text { (Heckel) }\end{array}$ & $\begin{array}{l}\text { Poeciliopsis retropinna } \\
\text { (Regan) }\end{array}$ & $\begin{array}{c}\text { Xiphophorus hellerii } \\
\text { Heckel }\end{array}$ & $\begin{array}{l}\text { Profundulus labialis } \\
\text { (Günther) }\end{array}$ \\
\hline & Río Danta ${ }^{\dagger}$ & Río Chiriquicito & Río Danta & Río Chicoasen \\
\hline Body length & $203(157-278 ; \mathrm{n}=7)[108(88-135 ; \mathrm{n}=7)]$ & $182(165-205 ; \mathrm{n}=9)$ & $111(95-127 ; \mathrm{n}=10)$ & $211(145-255 ; \mathrm{n}=8)$ \\
\hline Greatest width & $70(42-90 ; n=4)[29(22-38 ; n=6)]$ & $60-96$ & $43(34-51 ; n=7)$ & 65 \\
\hline Pharynx & $12(10-15 ; \mathrm{n}=3)[8]$ & 13 & - & - \\
\hline Haptor width & $62(48-72 ; \mathrm{n}=9)[46(40-57 ; \mathrm{n}=5)]$ & $64(57-70 ; n=12)$ & $30-38$ & $58(55-60 ; n=8)$ \\
\hline Ventral anchor length & $27(25-29 ; \mathrm{n}=20)[25(25-26 ; \mathrm{n}=8)]$ & $27(26-28 ; n=25)$ & $21(20-23 ; \mathrm{n}=33)$ & $23(22-25 ; \mathrm{n}=20)$ \\
\hline Base width & $11(10-12 ; \mathrm{n}=12)[(11-12)]$ & $11(10-12 ; \mathrm{n}=12)$ & $11(10-12 ; \mathrm{n}=12)$ & $12(11-13 ; \mathrm{n}=15)$ \\
\hline Dorsal anchor length & $19(18-22 ; \mathrm{n}=12)[(17-18)]$ & $20(19-21 ; \mathrm{n}=13)$ & $17(16-17 ; \mathrm{n}=23)$ & $19(18-20 ; \mathrm{n}=21)$ \\
\hline Base width & $10(9-10 ; \mathrm{n}=7)$ & $10(\mathrm{n}=6)$ & $9(7-10 ; n=9)$ & $10(10-12 ; \mathrm{n}=15)$ \\
\hline Ventral bar length & $30(26-34 ; \mathrm{n}=10)[27(25-28 ; \mathrm{n}=3)]$ & $29(28-30 ; n=9)$ & $21(18-24 ; n=9)$ & $23(21-24 ; n=3)$ \\
\hline Dorsal bar length & $25(22-28 ; \mathrm{n}=6)$ & $22-23$ & $17(15-20 ; n=3)$ & $21-22$ \\
\hline Hook pair 1 & $11(9-13 ; n=4)$ & $12-13$ & $10(10-11 ; \mathrm{n}=6)$ & $12(11-13 ; n=6)$ \\
\hline Hook pair 5 & $10(\mathrm{n}=2)$ & - & $15-17$ & $11(11-12 ; \mathrm{n}=3)$ \\
\hline Hook pairs $2-4,6$ and 7 & $15(14-17 ; n=15)$ & $17(17-18 ; n=6)$ & $16(15-17 ; n=4)$ & $17(15-19 ; n=5)$ \\
\hline Ring diameter of $\mathrm{MCO}$ & $13(11-15 ; n=3)$ & $20(17-23 ; n=6)$ & - & $14(13-17 ; \mathrm{n}=9)$ \\
\hline Germarial length & $30(29-32 ; n=3)$ & - & - & - \\
\hline Germarial width & $16(14-18 ; n=3)$ & - & - & - \\
\hline Testis width & 9 & - & - & - \\
\hline Vaginal sclerite length & $15(13-16 ; n=10)$ & $15(12-18 ; n=5)$ & $15(14-18 ; \mathrm{n}=17)$ & $16(15-18 ; \mathrm{n}=10)$ \\
\hline
\end{tabular}

* type host; $\uparrow$ type locality; small forms of $U$. vaginoclaustroides $\mathrm{sp} . \mathrm{n}$. are in brackets.

rinodontiformes: Poeciliidae) from Río Danta $\left(16^{\circ} 09^{\prime} 08^{\prime \prime} \mathrm{N}\right.$; $90^{\circ} 54^{\prime} 06^{\prime \prime} \mathrm{W} ; 200 \mathrm{~m}$ ), a tributary of the Río Lacantún in the BRMA, Chiapas, Mexico; 4/9 (mean TL 62) (44\%), 6.25, June 2012. Profundulus labialis from Río at rancho San Antonio, Chicoasén, Chiapas, México (16 $58^{\prime} 31^{\prime \prime N}$; 9303'45"W; 375 m), 6/20 (mean TL 39 mm) (30\%), 7.3, 2-18, May 2013.

Honduras: Profundulus portillorum Matamoros et Shaefer from Creek at Ojojona, Fco. Morazán (Río Nacaome basin) (135' $\left.44^{\prime \prime} \mathrm{N} ; 8^{\circ} 17^{\prime} 40^{\prime \prime} \mathrm{W} ; 1391 \mathrm{~m}\right), 18 / 30$ (mean TL $\left.49 \mathrm{~mm}\right)$ (60\%), 9, May 2014. Lepaterique, Fco. Morazán (Río Na-

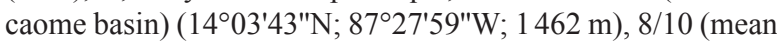
TL $56 \mathrm{~mm}$ ) (80\%), 6.6, May 2014.

Site of infection: Gill lamellae.

Specimens deposited: 25 vouchers in CNHE (9861).

Previous records: Gills of the introduced $X$. hellerii (type host) in India (Jogunoori et al. 2004) and Los Berros spring in the state of Durango, northern Mexico (Mendoza-Palmero and Aguilar-Aguilar 2008).

Remarks. Present specimens of $U$. vaginoclaustrum from $X$. hellerii and Profundulus labialis do not differ significantly from the type specimens examined (three paratypes - BMNH 2003.9.14.13-20). This species was originally described from introduced $X$. hellerii in India (Jogunoori et al. 2004). Although the specimens from Mexico are similar in shape, they are smaller (mainly in body length) than those used in the original description (i.e. 95-127 $\mu \mathrm{m} v s$ 168-244 $\mu \mathrm{m}$ long). Mendoza-Palmero and Aguilar-Aguilar (2008) also reported smaller specimens based on the size of anchors/bars of $U$. vaginoclaustrum collected from introduced $X$. hellerii in northern Mexico. This size difference might be due to intraspecific variation, probably attributed to different environmental factors in different locations (i.e. Mexico vs India).

\section{DISCUSSION}

This study not only provides morphological descriptions of two new species of Urocleidoides, but also shows the geographical distributions of these monogenoids on 9 species of Profundulus (P. balsanus, P. guatemalensis, P. kreiseri, P. labialis, P. oaxacae, $P$. portillorum, $P$. punctatus, Profundulus sp. 1, and Profundulus sp. 2) and 3 species of Poeciliidae ( $P$. bimaculata, P. retropinna and $X$. hellerii). Only Profundulus hildebrandi Miller and Profundulus candalarius (Hubbs) from Chiapas, Mexico were negative for monogenoids. Urocleidoides is unique within the tropics by containing 20 species (plus two new ones described in this study) parasitising fishes of 10 families from 3 orders (Characiformes, Cyprinodontiformes and Gymnotiformes) (see Mizelle and Price 1964, Kritsky et al. 1986, Moreira et al. 2015). Despite the fact there are no phylogenetic analyses of species that parasitise fish, it is clearly composed by different taxonomic groups, suggesting complex origins.

As described in this study, $U$. vaginoclaustroides is phenotypicly similar $U$. vaginoclaustrum, both parasitising poeciliids (P. bimaculata, $P$. retropinna and $X$. hellerii) and a profundulid, $P$. labialis (Profundulidae). The ocurrence of $U$. simonae, $U$. vaginoclaustroides and $U$. vaginoclaustrum on profundulids and poeciliids documented here extends the known geographical distribution of species of Urocleidoides (sensu stricto) to southeastern Mexico. Additionally, $U$. vaginoclaustrum was collected on $X$. hellerii in their native habitat (i.e. the BRMA) in Mexico, which contrast with the previous records of this species on introduced populations of $X$. helleri in the north of Mexico, out of its natural range of distribution (Mendoza-Palmero and Aguilar-Aguilar 2008). 
Among fish species reported as hosts of Urocleidoides are those of the order Characiformes (200 species in Africa and more than 1200 species in the Neotropics in about 14-16 families) and Cyprinodontiformes (850 species in about 110 genera), which comprise the most speciose assemblages of fishes in the tropics and North America (Costa 1998). The mixing of fish (i.e. different taxonomic groups occurring in sympatry) with different origins and evolutionary trajectories have resulted in groups with different rates of diversification in the tropics (Morris et al. 2001, Chen and Borowsky 2004).

Given this scenario, interactions of potential host species for Urocleidoides may have been an important factor on distribution of these parasite species resulting in different host switching events and speciation. Additional morphological and molecular data are necessary to identify patterns of diversification among profundulid and poeciliid species and their respective monogenoids. This will allow us to explore whether host phylogeny and/or geographic distribution are important factors in driving diversification of these ectoparasites.

Acknowledgements. This study was supported by the Consejo Nacional de Ciencia y Tecnología (CONACyT) Mexico (GSM grant no. 155372). Data from the Biosphere Reserve of Montes Azules (BRMA), Chiapas, Mexico were obtained in the frame of the project "Conservación, manejo y restauración de los ecosistemas acuáticos del Río Lacantún”, promoted and coordinated by the Centro Interdisciplinario de Biodiversidad y Ambiente (CEIBA), A. C. with financing from Natura y Ecosistemas Mexicanos, A. C. Thanks are due to Gisela Reina and Fernando Alda for providing parasites from P. retropinna collected for the Barcoding project "Estimating diversity of Neotropical monogenean (Platyhelminthes) fish parasites" with financial support from the Smithsonian Tropical Research Institute (STRI) of the Republic of Panama. We are also very grateful to all local governmental agencies for issuing collection permits as well as Olga Contreras (Guatemala), Samuel Álvarez Calderón, Enrique Barraza and Nestor Herrera (El Salvador), and María Eugenia Mondragón and Diego Ardon (Honduras) for assistance during field work.

\section{REFERENCES}

Bush A.O., Lafferty K.D., Lotz J.M., Shostak A.W. 1997 Parasitology meets ecology on its own terms: Margolis et al. revisited. J. Parasitol. 83: 575-583.

Costa, W.J.E.M. 1998: Phylogeny and classification of the Cyprinodontiformes (Euteleostei: Atherinomorpha): a reappraisal. In: L.R. Malabarba, R.E. Reis, R.P. Vari, Z.M. Lucena and C.A.S. Lucena (Eds.), Phylogeny and Classification of Neotropical Fishes. Edipurcs, Porto Alegre, pp. 537-560.

Chen K.-C., Boroswsky R.L. 2004: Comparative phylogeography of Xiphophorus variatus and Heterandria jonesi (Poeciliidae) using RAPD data. Ichthyol. Explor. Freshwaters 15: 25-40.

Doadrio I., Carmona J.A., Martínez E., De Sostoa A. 1999 Genetic variation and taxonomic analysis of the subgenus Profundulus. J. Fish Biol. 55: 751-766.

Jogunoori W., Kritsky D.C., Venkatanarasaiah J. 2004: Neotropical Monogenoidea. 46. Three new species from the gills of introduced aquarium fishes in India, the proposal of Heterotylus n. g. and Diaphorocleidus n. g., and the reassignment of some previously described species of Urocleidoides Mizelle \& Price, 1964 (Polyonchoinea: Dactylogyridae). Syst. Parasitol. 58: $115-124$.

Kritsky D.C., Thatcher V.E., Boeger W.A. 1986: Neotropical. 8. Revision of Urocleidoides (Dactylogyridae, Ancyrocephalinae). Proc. Helminthol. Soc. Wash. 53: 1-37.

Mendoza-Franco E.F, Caspeta-Mandujano J.M., SalgaDo-Maldonado G. 2013: New species of Cacatuocotyle (Monogenoidea, Dactylogyridae) parasitizing the anus and the gill lamellae of Astyanax aeneus (Pisces, Ostariophysi: Characi- dae) from the Rio Lacantún basin in the Biosphere Reserve of Montes Azules, Chiapas, Mexico. Parasitol. Res. 112: 199-205.

Mendoza-Franco E.F., Vidal-Martínez V.M. 2011: First records of known endoparasitic species of Pseudempleurosoma Yamaguti, 1965 (Monogenoidea: Dactylogyridae) from tetraodontid and rachycentrid fish from the northern coast of the $\mathrm{Yu}-$ catan, Peninsula, Mexico. J. Parasitol. 94: 1020-1025.

Mendoza-Palmero C.A., Aguilar-Aguilar R. 2008: Record of Urocleidoides vaginoclaustrum Jogunoori, Kritsky and Venkatanarasaiah, 2004 (Monogenea: Dactylogyridae) from a freshwater fish in Mexico. Parasitol. Res. 103: 1235-1236.

Mizelle J.D., Price C.E. 1964: Studies on monogenetic trematodes. XXVII. Dactylogyrid species with the proposal of Urocleidoides gen. n. J. Parasitol. 50: 579-584.

Moreira J., Scholz T., LuQue J.L. 2015: First data on the parasites of Hoplias aimara (Characiformes): description of two new species of gill monogeneans (Dactylogyridae). Acta Parasitol. 60: 254-260.

Morris M.R., Queiroz K., Mozirot D.C. 2001: Phylogenetic relationships among populations of northern swordtails (Xiphophorus) as inferred from allozyme data. Copeia 1: 65-81.

Salgado-Maldonado G., Matamoros W.A., Caspeta-Mandujano J.M., Martínez-Ramírez E., Mendoza-Franco E.F., Velázquez-Velázquez E. 2014: Range extension of helminth parasites of Profundulus spp. (Teleostei: Profundulidae) from Southern Mexico and Central America. Check List 6: 1507-1513. 